\title{
Prevalence and risk factors for non- alcoholic fatty liver in children and youth with obesity
}

Carolina Jimenez-Rivera ${ }^{1,5^{*}}$ (D), Stasia Hadjiyannakis ${ }^{2,5}$, Jorge Davila ${ }^{3,5}$, Julie Hurteau ${ }^{3,5}$, Mary Aglipay 4,5 , Nick Barrowman ${ }^{4,5}$ and Kristi B. Adamo ${ }^{6}$

\begin{abstract}
Background: Non- Alcoholic Fatty Liver (NAFL) is a spectrum of liver diseases (LD) that ranges from benign fatty infiltration of the liver to cirrhosis and hepatic failure. Hepatic ultrasound (US) and serum alanine aminotransferase (ALT) are often used as markers of NAFL. Our aim is to describe prevalence of NAFL and associated findings on ultrasound (US) and biochemical parameters in a population of children and adolescents with obesity at the Children's Hospital of Eastern Ontario.

Methods: Children with Obesity (BMI >95th percentile) ages 8-17 years presenting to the Endocrinology and Gastroenterology clinics, without underlying LD were prospectively recruited from 2009 to 2012. Fasting lipid profile, HOMA IR) and serum adiponectin levels were measured. NAFL was defined as ALT $>25$ and $>22 \mathrm{IU} / \mathrm{mL}$ (males and females respectively) and/or evidence of fatty infiltration by US. Logistic regression was performed to assess associations.

Results: 97 children with obesity included in the study (Male 43\%). Mean age was $12.9 \pm 3.2$ years (84\% were older than 10 y). Mean BMI-Z score was $3.8 \pm 1$.4. NAFL was identified in $85 \%(82 / 97)$ of participants. ALT was elevated in $61 \%$ of patients. Median triglyceride (TG) level was higher in children with NAFL(1.5 \pm 0.9 vs. $1.1 \pm 0.5 \mathrm{mmol} / \mathrm{L}$, $p=0.01)$. Total cholesterol, HDL, LDL and Non HDL cholesterol were similar in both groups $(p=0.63, p=0.98$, $p=0.72$ and $p=0.37$ respectively). HOMA IR was $\geq 3.16$ in $53 \%$ of children( $55 \%$ in those with NAFL and $40 \%$ in those without NAFL). Median serum adiponectin was $11.2 \mu \mathrm{g} / \mathrm{ml}(\mathrm{IQR} 7.3-18.3)$ in children with NAFL vs. $16.1 \mu \mathrm{g} /$ ml(IQR 9.0-21.9) in those without NAFL( $p=0.23)$. Liver US was reported as normal in $30 \%$, mild fatty infiltration in $38 \%$, moderate in $20 \%$ and severe in $12 \%$. TG were significantly higher $(1.5 \mathrm{mmol} / \mathrm{L} \mathrm{vs} .1 .0 \mathrm{mmol} / \mathrm{L}, p<0.01)$ and $\mathrm{HDL}-\mathrm{C}$ was lower $(1.0 \mathrm{mmol} / \mathrm{L}$ vs. $1.1 \mathrm{mmol} / \mathrm{L}, p=0.05)$ in children with moderate and severe NAFL by US. BMI-Z score, HOMA IR, serum adiponectin and HDL levels were not associated with NAFL, however TG were significantly associated(OR $=3.22(95 \% \mathrm{Cl}: 1.01-10.25, p=0.04)$ ).
\end{abstract}

Conclusion: NAFL is highly prevalent in obese children and youth. Elevated TG levels are associated with NAFL; these findings may serve as a noninvasive screening tool to help clinicians identify children with obesity needing liver biopsy and/or more aggressive therapeutic interventions.

Keywords: Non-alcoholic fatty liver, Obesity, Dyslipidemia

\footnotetext{
* Correspondence: cajimenez@cheo.on.ca

'Division of Gastroenterology, Hepatology and Nutrition, University of

Ottawa, Ottawa, Canada

${ }^{5}$ Children's Hospital of Eastern Ontario, Faculty of Health Sciences, University

of Ottawa, Ottawa, Canada

Full list of author information is available at the end of the article
} International License (http://creativecommons.org/licenses/by/4.0/), which permits unrestricted use, distribution, and reproduction in any medium, provided you give appropriate credit to the original author(s) and the source, provide a link to the Creative Commons license, and indicate if changes were made. The Creative Commons Public Domain Dedication waiver (http://creativecommons.org/publicdomain/zero/1.0/) applies to the data made available in this article, unless otherwise stated. 


\section{Background}

Childhood obesity is one of the primary predictors of obesity in adults; more than two thirds of children with obesity will become obese adults [1-3]. Non-alcoholic fatty liver (NAFL), metabolic syndrome, type 2 diabetes mellitus, obstructive sleep apnea and cardiovascular disease are not only well-described complications of obesity in adults, but are also becoming increasingly recognized conditions in children.

NAFL disease is a clinical pathological condition characterized by broad spectrum of liver damage ranging from isolated steatosis to steatohepatitis and cirrhosis [4]. The prevalence of NAFL in obese children is not well known. A very wide range of prevalence has been reported in the literature. Ten to $77 \%$ of children with obesity have evidence of hepatic fatty infiltration by ultrasound and $25 \%$ of them have elevation of liver enzymes $[5,6]$. Children with NAFL are usually asymptomatic, they may present with vague abdominal pain and incidental elevation of alanine aminotransferase (ALT) screened due to child's body habitus [7, 8]. NAFL is uncommon in children under 8 years of age, the average age at presentation is 12 years [9]. Liver biopsy is the gold standard for diagnosing NAFL, nonetheless performing a liver biopsy is invasive and carries risks of complications and often requires patient's admission post procedure $[10,11]$. The aim of this study is to identify the prevalence of fatty liver by non-invasive methods in a pediatric population with obesity as well as evaluate risk factors for NAFL by associations with ultrasound (US) findings and biochemical parameters.

\section{Methods}

Children 8-17 years old with obesity (Body Mass Index, BMI > 95th percentile for age and sex) were prospectively recruited from September 2009 and December 2012. The study was conducted at the Children's Hospital of Eastern Ontario, a tertiary care academic center, affiliated with the University of Ottawa. Institutional Research Board approval was obtained to conduct this study. Written informed consent was obtained from parent/legal guardian of children under 16 years of age; children 16 years and above provided their own consent.

Body weight was measured using a balance beam scale, calibrated to the nearest $0.1 \mathrm{~kg}$ daily. Height was measured using a SECA stadiometer. BMI was calculated according to the following formula: $\mathrm{BMI}=$ weight in $\mathrm{kg} /$ height in metres [2]. A BMI z score was calculated using https://www.cdc.gov/growthcharts/ norms.

A baseline blood sample was obtained for fasting glucose, insulin, lipid profile including total cholesterol, low-density lipoprotein cholesterol (LDLC), triglycerides (TG), high-density lipoprotein cholesterol (HDLC), alanine aminotransferase and aspartate aminotransferase
(ALT and AST respectively) and serum adiponectin. ALT $>25 \mathrm{IU} / \mathrm{L}$ was considered abnormal in boys and $>22$ IU/L in girls [12]. Other causes of liver diseases were excluded in children with elevated liver enzymes by screening for hepatotropic viruses (hepatitis A, B and C, cytomegalovirus and Epstein Barr virus), alpha 1 antitrypsin (A1AT) levels as well as autoantibodies and immunoglobulin $\mathrm{G}$ to rule out A1AT deficiency and autoimmune hepatitis respectively. Patients were not included in the study if there was a history of alcohol intake or had any other type of liver diseases such as drug induced fatty liver and genetic disorders.

Insulin resistance was estimated using the Homeostasis Model Assessment (HOMA) formula, which calculates insulin resistance based on simultaneous fasting blood glucose and insulin levels [13]. The HOMA formula is calculated as fasting insulin $(\mathrm{mU} / \mathrm{ml})$ multiplied by fasting plasma glucose $(\mathrm{mmol} / \mathrm{L})$ divided by 22.5 . Lower HOMA values indicate higher insulin sensitivity. The estimate obtained with HOMA correlates well $(r=-0.91, p<0.001)$ with measures of insulin resistance obtained from obese and non-obese children and adolescents with the use of the euglycemic-hyperinsulinemic clamp technique [14]. HOMA > 3.16 was considered a marker of insulin resistance as described by Keskin, et al. [15]. Serum high molecular weight adiponectin was measured in duplicate with the MILLIPLEX MAP Panel A kit (Millipore, Billerica, MA) using Luminex xMAP technology as described by the manufacturer. Under our experimental conditions, the intra- and inter-assay CV were both $<10 \%$.

Hepatic fatty infiltration: Abdominal US determined the brightness of the liver echogenicity compared with the kidney, portal vein wall and diaphragm [16], to assess the degree and severity of fatty liver. All US were performed by one of the two radiologists involved in the study and reviewed separately by both radiologists (JD and $\mathrm{JH}$ ). The radiologists were blinded to the blood test-results and clinical history of patients. In the event of discrepancy they discussed the findings and came to an agreement. A General Electric Logiq $9^{\circ}$ machine was used in all cases (GE Healthcare, Quebec, Canada). Axial and sagittal images through the liver were acquired using a $5 \mathrm{MHz}$ probe. A sagittal image in the mid-axillary plane including the liver and right kidney was obtained to assess the echogenicity of the liver in comparison to the kidney. Axial right paramedian images were used to assess the degree of echo penetration through the liver, the clarity of portal vessels and visualisation or the diaphragm. Depths, focus point, 2D gain, were kept constant for all studies as starting parameters. If the deep portions and vessels of the liver were not seen, then imaging parameters were adjusted and the images were repeated. Standard ultrasound scoring system identified children without signs of liver 
steatosis and those with different degrees of steatosis including mild, moderate and severe fatty infiltration.

Children were excluded if they had known underlying liver disease, and/or had an insurmountable language barrier that would prevent consent to participation.

NAFL was defined as a mild, moderate or severe fatty infiltration as demonstrated by liver US or ALT levels of $>25 \mathrm{IU} / \mathrm{L}$ in males and $>22 \mathrm{IU} / \mathrm{L}$ in females. Descriptive statistics were calculated for all patient characteristics. Univariate associations between NAFL and each potential risk factors including TG, HDLC, non HDLC, HOMA IR, total cholesterol, LCLC, BMI $\mathrm{z}$ score and serum adiponectin, were assessed using Student t-tests, Mann-Whitney U tests, or Pearson chi-squared tests where appropriate. Univariate logistic regression was also used to determined estimates of effect and respective confidence intervals for each covariate. Variables were log-transformed as appropriate in the univariate logistic regression. To evaluate the sensitivity and specificity of different thresholds of TG as a potential screening tool for NAFL, an ROC curve was constructed. The area under the ROC curve was computed, together with a $95 \%$ confidence interval. All analyses were completed using SPSS version 23 and $\mathrm{R}$ version 3.0.2,

\section{Results}

A total of 98 children consented to participate and 97 were included in the study period, one patient was excluded due to incomplete biochemical test results. Of those, 42 (43\%) were males. Mean age was $12.9 \pm 3.2$ years. Mean BMI-Z score was $3.8 \pm 1.4$. Median ALT was 26.0 IU/L (IQR 17.0-33.5); abnormal ALT was found in 59/98 (60.8\%) children. Median AST was $26.5 \mathrm{IU} / \mathrm{L}$ (21.0-30.0). Investigations for underlying liver disease were negative. Patient characteristics are shown in Table 1.

There were 9 patients (out of 83 with available data) receiving medications, 2 were on oral corticosteroids, one of which was also receiving metformin, one on concomitant lowering lipid agent and metformin, one on both insulin and metformin, one on inulin alone and 4 on metformin alone.

Median TG level was $1.3 \mathrm{mmol} / \mathrm{L}$ (IQR 0.8-1.9), median HDL-C was $1.0 \mathrm{mmol} / \mathrm{L}$ (IQR 0.9-1.2), median LDLC was $2.50 \mathrm{mmol} / \mathrm{L}(2.1-3.1)$ median HOMA IR was 3.3 (IQR 2.0-5.8) and median serum adiponectin was $11.3 \mu \mathrm{g} / \mathrm{ml}$ (IQR 7.5-19.2). Elevated triglycerides were associated with NAFL in simple logistic regression $(\mathrm{OR}=3.22,95 \% \mathrm{CI}=1.01-10.25, p=0.01)$. BMIZ score, HOMA IR, serum adiponectin and HDL levels were not associated with NAFL. Figure 1 shows odds ratios and associated 95\% confidence intervals. There were no statistically significant associations between NAFL and
Table 1 Patient characteristics

\begin{tabular}{|c|c|c|}
\hline & $N$ & \\
\hline Age, mean (SD) & 97 & $12.9(3.2)$ \\
\hline Age- Pubertal (10+ years), $n(\%)$ & 97 & $81(83.5)$ \\
\hline Male, $n(\%)$ & 97 & $42(43.3)$ \\
\hline \multicolumn{3}{|l|}{ Race, $n(\%)$} \\
\hline White & 80 & $66(82.5)$ \\
\hline Black & 80 & $6(7.5)$ \\
\hline Arab & 80 & $6(7.2)$ \\
\hline Aboriginal & 80 & $4(5)$ \\
\hline South Asia & 80 & $3(3.8)$ \\
\hline BMI z score, mean (SD) & 97 & $3.8(1.4)$ \\
\hline \multicolumn{3}{|l|}{ Comorbidities, $n(\%)$} \\
\hline Type 2 diabetes & 84 & $0(0.0)$ \\
\hline NAFLD & 84 & $49(58.3)$ \\
\hline Sleep apnea & 83 & $14(16.9)$ \\
\hline Pre-existing acanthosis nigricans & 79 & $44(55.7)$ \\
\hline \multicolumn{3}{|l|}{ Blood levels } \\
\hline Total Cholesterol (mmol/L), mean (SD) & 97 & $4.4(0.8)$ \\
\hline Triglycerides (mmol/L), median (IQR) & 96 & $1.3(0.8-1.9)$ \\
\hline HDLC (mmol/L), median (IQR) & 97 & $1.0(0.9-1.2)$ \\
\hline LDLC, mean (SD) & 96 & $2.6(0.7)$ \\
\hline Non HDL Cholesterol (mmol/L), mean (SD) & 84 & $3.3(0.8)$ \\
\hline HOMAIR +3.16, n(\%) & 95 & $50(52.6)$ \\
\hline ALT (IU/mL), median (IQR) & 97 & $26.0(17.0-33.5)$ \\
\hline AST(IU/mL), median (IQR) & 95 & $26.0(21.0-30.0)$ \\
\hline Adiponectin, median (IQR) & 96 & $11.3(7.5-19.3)$ \\
\hline NAFLD grading by US, $n(\%)$ & 97 & \\
\hline Normal & & $29(29.9)$ \\
\hline Mild & & $37(38.1)$ \\
\hline Moderate & & 19 (19.6) \\
\hline Severe & & $12(12.4)$ \\
\hline
\end{tabular}

$N$ number of patients with available data, BMI Body Mass Index, NAFLD NonAlcoholic Fatty Liver Disease, HDLC High Density Lipoprotein Cholesterol, LDLC Low Density Lipoprotein Cholesterol, HOMA IR Homeostatic Model Assessment Insulin Resistance, AST Aspartate aminotransferase, ALT Alanine aminotransferase

AST, HDLC, HOMAIR, sex, non HDL cholesterol, total cholesterol, BMI Z score, LDLC, age or adiponectin. However, there was a significant association between TG and NAFL (OR $=3.22$ (95\% CI: $1.01-10.25), p=0.04)$. To evaluate the sensitivity and specificity of TG as a potential screening tool for NAFL, an ROC curve was constructed. The area under the ROC curve was 0.69 (95\% CI 0.54 to 0.84). An example screening cut off of TG > $=0.755$ gives a sensitivity of $90 \%$ and a specificity of $40 \%$ for predicting NAFL.

Additional analysis was performed to assess whether risk factors could be subsequently identified when US detected moderate and severe fatty infiltration in 


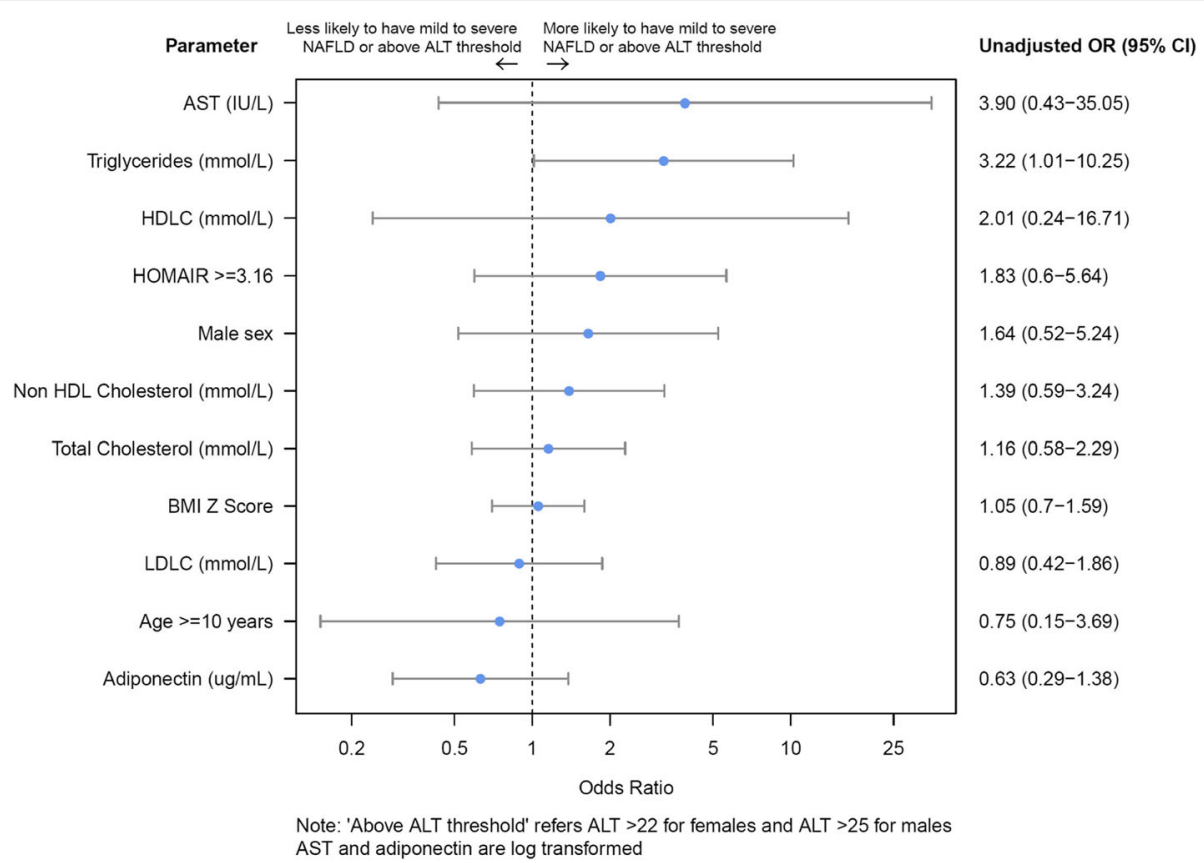

Fig. 1 Forest graph showing Odds ratio for ALL patients with NAFL. NAFL Non-Alcoholic Fatty Liver, AST aspartate aminotransferase, HDLC High Density Lipoprotein Cholesterol, HOMA IR Homeostatic Model Assessment Insulin Resistance, LDLC Low Density lipoprotein Cholesterol, BMI Body Mass Index

combination with an abnormal ALT (Fig. 2). Once again TG were significantly higher in children with NAFL $(1.5 \mathrm{mmol} / \mathrm{L}$ vs. $1.0 \mathrm{mmol} / \mathrm{L}, p<0.01)$.

Liver US showed some degree of steatosis in $70 \%$ (69/98) of the cohort, varying from mild fatty infiltration in 38\% (37/97), moderate in 20\% (19/97) and severe 12\% (12/97). Liver US was reported as normal in $30 \%(29 / 97)$ of children. Fourteen children with normal US had abnormal ALT (14/29, 48.3\%). Two thirds of children with some degree of fatty infiltration by US had elevated ALT $(45 / 68,66.2 \%)$. Prevalence of NAFL (elevated ALT and US showing fatty infiltration)

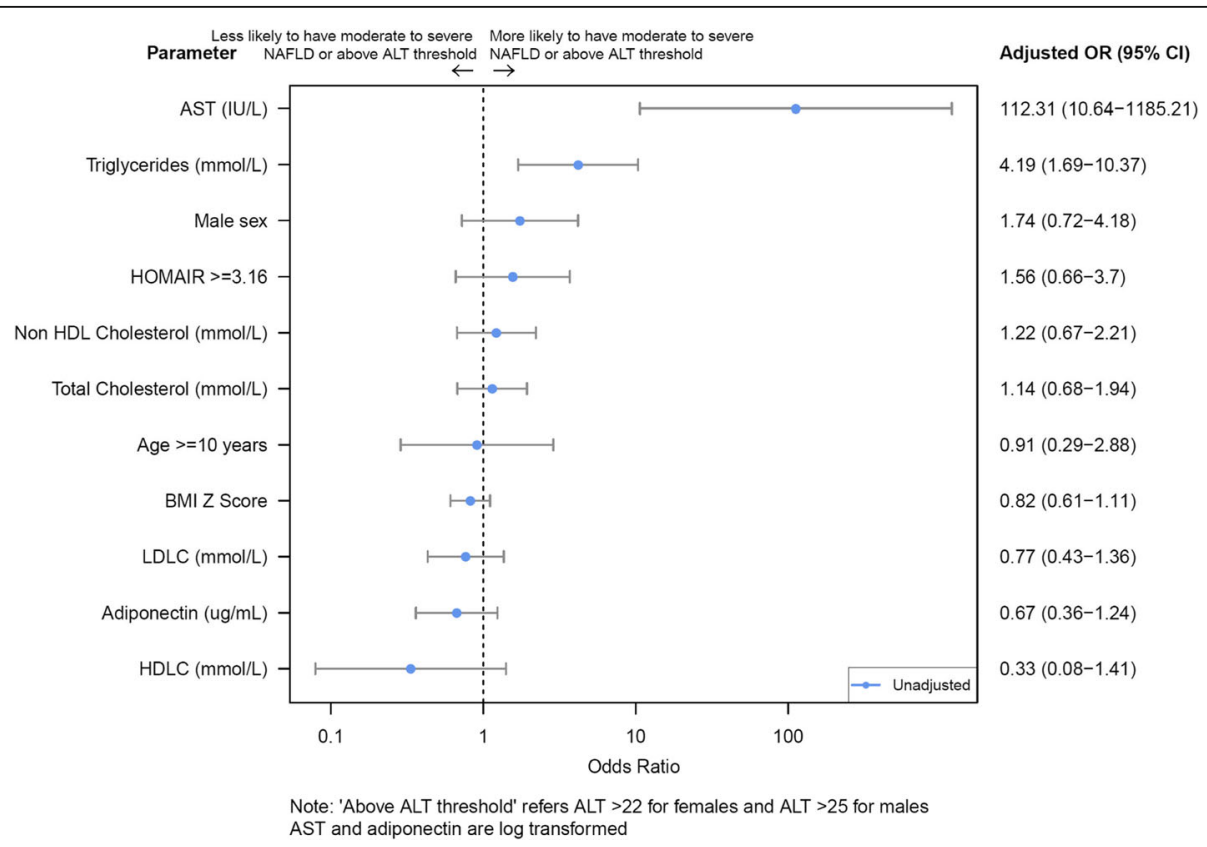

Fig. 2 Forest graph for moderate to severe NAFL. NAFL Non-Alcoholic Fatty Liver, AST aspartate aminotransferase, HDLC High Density Lipoprotein Cholesterol, HOMA IR Homeostatic Model Assessment Insulin Resistance, LDLC Low Density lipoprotein Cholesterol, BMI Body Mass Index 
was $85 \%$ (82/97). Table 2 shows univariate associations between NAFL and selected risk factors.

\section{Discussion}

Childhood obesity is the main risk factor for the development of NAFL. Suspicion of NAFL is often based on patient's body habitus, which may prompt clinicians to investigate further by assessing hepatic steatosis by liver US and measurement of ALT. Liver biopsy is the ideal test to establish the diagnosis [17], however this is invasive and access may be limited.

The prevalence of NAFL in pediatric patients with obesity by the definition used in this study was high (85\%). Our finding was very similar to Xanthakos, et al. [18], where the prevalence of NAFL was $83 \%$ based on liver biopsies taken at the time of bariatric surgery. Another study [19] reported lower prevalence calculated between 20 and 30\% when abnormal transaminases and ultrasound were used respectively, this may be related to a different definition of abnormal transaminases.

In the logistic regression analysis, it appeared TG levels were associated to NAFL. Given the broad confidence interval found, the association may or may not have clinical impact and it should be taken with caution. These findings were also demonstrated in a similar study by Navarro-Jarabo, et al. [20], where a higher level of triglycerides and higher BMI were related to hepatic steatosis. In addition to this, we looked more closely at children with moderate to severe steatosis by US and we found that HDLC was lower in this group of patients, which can be related to insulin resistance [21]. A recent study by Lee et al. [22], described the association between NAFL detected by US and hypertriglyceridemia among other biochemical and anthropometric parameters such as weight to height ratio and waist circumference amongst others.

Adiponectin, an adipocyte secreted protein, has been recognized for its physiological role in decreasing postprandial rise of free fatty acids and enhancement of insulin signalling (inhibits lipolysis in adipose tissue, stimulated free fatty acid oxidation in hepatocytes, stimulates insulin action in hepatocytes, inhibits gluconeogenesis, and improves insulin mediated glucose transport in skeletal muscle) [23]. Although secreted by the adipocyte, paradoxically adiponectin is reduced in obese adults [24], increased in anorexia nervosa and during periods of weight loss $[25,26]$. There is a growing body of literature related to its role in the development of NAFL [23]. Since low adiponectin level was an indicator of NAFL in a large population of adults [27], we measured levels of adiponectin in our pediatric sample however they did not correlate with the degree of hepatic steatosis. Given "normal" adiponectin ranges have not been established and standardized in children

Table 2 Univariate Associations between NAFLD definition and selected covariates

\begin{tabular}{|c|c|c|c|c|}
\hline & \multirow[t]{2}{*}{$N$} & \multicolumn{2}{|c|}{$\begin{array}{l}\text { Meets NAFLD definition: Any (mild/moderate/severe) fatty infiltration } \\
\text { or } A L T>25 \mathrm{IU} / \mathrm{mL} \text { (males)or ALT }>22 \mathrm{IU} / \mathrm{mL} \text { (females) }\end{array}$} & \multirow[t]{2}{*}{$P$ Value } \\
\hline & & No & Yes & \\
\hline Sex, $n(\%)$ & & & & 0.57 \\
\hline Male & 42 & $5(11.9)$ & $37(88.1)$ & \\
\hline Female & 55 & $10(18.2)$ & $45(81.8)$ & \\
\hline Age (category), $n$ (\%) & & & & 1.00 \\
\hline$<10$ Years & 16 & $2(12.5)$ & $14(87.5)$ & \\
\hline$>=10$ Years & 81 & $13(16.0)$ & $68(84.0)$ & \\
\hline Total Cholesterol (mmol/L), mean (SD) normal & 97 & $4.3(0.6)$ & $4.4(0.8)$ & 0.63 \\
\hline Triglycerides (mmol/L), mean (SD) & 96 & $1.1(0.5)$ & $1.5(0.9)$ & 0.01 \\
\hline HDLC (mmol/L), median (IQR) & 97 & $1.0(1.0-1.3)$ & $1.0(0.9-1.2)$ & 0.98 \\
\hline LDLC (mmol/L), mean (SD) normal & 96 & $2.7(0.6)$ & $2.6(0.8)$ & 0.72 \\
\hline Non HDL Cholesterol (mmol/L), mean (SD) & 84 & $3.1(0.6)$ & $3.3(0.8)$ & 0.37 \\
\hline HOMAIR, $n(\%)$ & 95 & & & 0.40 \\
\hline$<3.16$ & & $9(20)$ & $36(80)$ & \\
\hline$>=3.16$ & & $6(12)$ & $44(88)$ & \\
\hline AST(IU/mL), median (IQR) & 95 & $23.5(21.0-26.3)$ & $27.0(21.0-31.5)$ & 0.18 \\
\hline Adiponectin, median (IQR) & 96 & $16.1(9.0-21.9)$ & $11.2(7.3-18.3)$ & 0.23 \\
\hline BMI Z Score, mean (SD) & 97 & $3.7(1.3)$ & $3.8(1.4)$ & 0.80 \\
\hline
\end{tabular}

NAFLD Non-Alcoholic Fatty Liver Disease, ALT Alanine Aminotransferase, HDLC High Density Lipoprotein Cholesterol, LDLC Low Density Lipoprotein Cholesterol, HOMA IR Homeostatic Model Assessment Insulin Resistance, AST Aspartate aminotransferase, BMI Body Mass Index. 
it is possible that our patient population of children with obesity already had low levels of adiponectin. Unfortunately we did not have a control group of children without obesity for comparison.

Ultrasound is accessible and serves as a screening tool to identify hepatic fatty infiltration. In our study we found that almost $70 \%$ of children with obesity had some degree of fatty infiltration detected by US, which is higher compared to other studies where near $50 \%$ of children with obesity had some degree of steatosis [28]. This group also compared the extent of steatosis in prepubertal and pubertal children and found that pubertal children had higher scores in fatty infiltration by US. The limitation of using US in obese children lies in the technical difficulties and lack of accurate measures due to patient's body habitus and subjectivity of the technique. While ultrasound is a good screening tool, there remains the possibility of false positive or false negative results until a more precise gradation is established. Other imaging modalities that have been used include computed tomography (CT) and magnetic resonance (MR).While MR has proven the most accurate test to assess liver fatty infiltration, this test is costly and not accessible in many institutions. The use of $\mathrm{CT}$, which is more accurate when diagnosing fatty liver compared to US [29] is not commonly used because of the radiation exposure. Furthermore, using spectroscopy to quantify the amount of fat and water in the liver is more precise than conventional MR images [30-32]. Transient elastography is a non-invasive test that assesses the degree of fibrosis in chronic liver diseases including NAFL [33, 34]. Despite the accuracy of such tests, cost and accessibility remain limiting factors for general practitioners outside of tertiary care facilities.

Liver biopsies are rarely done in pediatric patients with obesity due to body habitus and procedure associated risks; however liver histology is considered the "gold standard" to establish the diagnosis of NAFL and we recognize this as a limitation in our study. A potential bias in our study was the inclusion of patients on medications that could affect metabolism of lipids and glucose; we elected to perform the analyses as the number of such patients was very small and findings on these patients did not differ from the entire cohort. Another limitation was the inability to pursue multiple logistic regression analyses due to the high prevalence of NAFL in our sample. As such, we cannot make conclusions about unconfounded associations in our study.

\section{Conclusion}

There is a high prevalence of NAFL in children and youth with obesity based on US findings and abnormal ALT. Additionally, elevated serum TG was an associated risk factor for NAFL. These findings may help clinicians identify high risk patients. Further efforts for effective interventions are required on this patient population as they are at a high risk of progressive liver disease in adulthood.

\begin{abstract}
Abbreviations
NAFL: Non-Alcoholic Fatty Liver; BMl: body mass index; ALT: alanine aminotransferase; AST: aspartate aminotransferase; HOMA: Homeostasis Model Assessment; LDL-C: low-density cholesterol; TG: triglycerides; HDLC: high-density cholesterol; CT: computed tomography; MR: magnetic resonance
\end{abstract}

\section{Acknowledgements \\ Not applicable.}

\section{Funding}

This study was funded through an academic Innovation Fund by the Government of Ontario. This entity did not have any influence in the development of the protocol, analyses, interpretation or writing of the manuscript.

\section{Availability of data and materials}

The datasets used and/or analyzed during the current study available from the corresponding author on reasonable request.

\section{Authors' contributions}

CJ-R: PI, collection and analysis of the data, literature search and writing of the manuscript. SH, recruitment of patients, analysis of data, contributions to manuscript writing. JD, performance of ultrasounds, data collection, contribution to manuscript. JH, performance of ultrasounds, data collection and writing of the manuscript. MA, statistical analysis of the data, contribution to the writing of the manuscript. NB, statistical analysis of the data, contribution to the writing of the manuscript. KA, performance of assays, analysis of data and contribution to the writing of the manuscript. All authors read and approved the final manuscript.

\section{Competing interests}

The authors declare that they have no competing interests.

\section{Consent for publication}

Not applicable.

\section{Ethics approval and consent to participate}

Children's Hospital of Eastern Ontario Research Ethics Board approval was obtained to conduct this study. Patients and/or parents consented to participate in the study.

\section{Publisher's Note}

Springer Nature remains neutral with regard to jurisdictional claims in published maps and institutional affiliations.

\section{Author details}

${ }^{1}$ Division of Gastroenterology, Hepatology and Nutrition, University of Ottawa, Ottawa, Canada. ${ }^{2}$ Division of Endocrinology and Metabolism, Faculty of Health Sciences, University of Ottawa, Ottawa, Canada. ${ }^{3}$ Diagnostic Imaging, University of Ottawa, Ottawa, Canada. ${ }^{4}$ Research Institute, University of Ottawa, Ottawa, Canada. ${ }^{5}$ Children's Hospital of Eastern Ontario, Faculty of Health Sciences, University of Ottawa, Ottawa, Canada. ${ }^{6}$ School of Human Kinetics, Faculty of Health Sciences, University of Ottawa, Ottawa, Canada.

Received: 11 November 2016 Accepted: 20 April 2017

Published online: 26 April 2017

References

1. Freedman DS, Srinivasan SR, Valdez RA, Williamson DF, Berenson GS. Secular increases in relative weight and adiposity among children over two decades: the Bogalusa heart study. Pediatrics. 1997;99(3):420-6.

2. Freedman DS, Khan LK, Dietz WH, Srinivasan SR, Berenson GS. Relationship of childhood obesity to coronary heart disease risk factors in adulthood: the Bogalusa heart study. Pediatrics. 2001;108(3):712-8. 
3. Magarey AM, Daniels LA, Boulton TJ, Cockington RA. Predicting obesity in early adulthood from childhood and parental obesity. Int J Obes Relat Metab Disord. 2003;27(4):505-13.

4. Brunt EM. Nonalcoholic steatohepatitis: definition and pathology. Semin Liver Dis. 2001;21(1):3-16.

5. Chan DF, Li AM, Chu WC, Chan MH, Wong EM, Liu EK, et al. Hepatic steatosis in obese Chinese children. Int J Obes Relat Metab Disord. 2004; 28(10):1257-63.

6. Schwimmer JB, McGreal N, Deutsch R, Finegold MJ, Lavine JE. Influence of gender, race, and ethnicity on suspected fatty liver in obese adolescents. Pediatrics. 2005;115(5):e561-5.

7. Manton ND, Lipsett J, Moore DJ, Davidson GP, Bourne AJ, Couper RT. Nonalcoholic steatohepatitis in children and adolescents. Med J Aust. 2000; 173(9):476-9.

8. Rashid M, Roberts EA. Nonalcoholic steatohepatitis in children. J Pediatr Gastroenterol Nutr. 2000;30(1):48-53.

9. Schwimmer JB, Burwinkle TM, Varni JW. Health-related quality of life of severely obese children and adolescents. JAMA. 2003 Apr 9;289(14):1813-9.

10. Harwood J, Bishop P, Liu H, Nowicki M. Safety of blind percutaneous liver biopsy in obese children: a retrospective analysis. J Clin Gastroenterol. 2010:44(10):e253-5.

11. Vajro P, Lenta S, Socha P, Dhawan A, McKiernan P, Baumann U, et al. Diagnosis of nonalcoholic fatty liver disease in children and adolescents: position paper of the ESPGHAN Hepatology committee. J Pediatr Gastroenterol Nutr. 2012;54(5):700-13.

12. Schwimmer JB, Dunn W, Norman GJ, Pardee PE, Middleton MS, Kerkar N, et al. SAFETY study: alanine aminotransferase cutoff values are set too high for reliable detection of pediatric chronic liver disease. Gastroenterology. 2010; 138(4):1357. -64, 1364

13. Gungor N, Saad R, Janosky J, Arslanian S. Validation of surrogate estimates of insulin sensitivity and insulin secretion in children and adolescents. J Pediatr. 2004;144(1):47-55.

14. Strauss RS, Pollack HA. Epidemic increase in childhood overweight, 1986-1998. JAMA. 2001;286(22):2845-8

15. Keskin M, Kurtoglu S, Kendirci M, Atabek ME, Yazici C. Homeostasis model assessment is more reliable than the fasting glucose/insulin ratio and quantitative insulin sensitivity check index for assessing insulin resistance among obese children and adolescents. Pediatrics. 2005:115(4):e500-3.

16. Yajima Y, Ohta K, Narui T, Abe R, Suzuki H, Ohtsuki M. Ultrasonographical diagnosis of fatty liver: significance of the liver-kidney contrast. Tohoku J Exp Med. 1983;139(1):43-50.

17. Schwimmer JB, Behling C, Newbury R, Deutsch R, Nievergelt C, Schork NJ, et al. Histopathology of pediatric nonalcoholic fatty liver disease. Hepatology. 2005;42(3):641-9.

18. Xanthakos S, Miles L, Bucuvalas J, Daniels S, Garcia V, Inge T. Histologic spectrum of nonalcoholic fatty liver disease in morbidly obese adolescents. Clin Gastroenterol Hepatol. 2006;4(2):226-32.

19. Guijarro de Armas MG, Monereo MS, Navea AC, Merino VM, Vega Pinero MB. Non-alcoholic fatty liver in children and adolescents with excess weight and obesity. Med Clin (Barc). 2015;144(2):55-8.

20. Navarro-Jarabo JM, Ubina-Aznar E, Tapia-Ceballos L, Ortiz-Cuevas C, PerezAisa MA, Rivas-Ruiz F, et al. Hepatic steatosis and severity-related factors in obese children. J Gastroenterol Hepatol. 2013;28(9):1532-8.

21. Elmaogullari S, Tepe D, Ucakturk SA, Karaca KF, Demirel F. Prevalence of Dyslipidemia and associated factors in obese children and adolescents. Clin Res Pediatr Endocrinol. 2015;7(3):228-34.

22. Lee $\mathrm{JH}$, Jeong $\mathrm{SJ}$. What is the appropriate strategy for diagnosing NAFLD using ultrasonography in obese children? World J Pediatr 2017.

23. Arita Y, Kihara S, Ouchi N, Takahashi M, Maeda K, Miyagawa J, et al. Paradoxical decrease of an adipose-specific protein, adiponectin, in obesity. Biochem Biophys Res Commun. 1999;257(1):79-83.

24. Pannacciulli N, Vettor R, Milan G, Granzotto M, Catucci A, Federspil G, et al. Anorexia nervosa is characterized by increased adiponectin plasma levels and reduced nonoxidative glucose metabolism. J Clin Endocrinol Metab. 2003;88(4):1748-52.

25. Andersen KK, Frystyk J, Wolthers OD, Heuck C, Flyvbjerg A. Gender differences of oligomers and total adiponectin during puberty: a cross-sectional study of 859 Danish school children. J Clin Endocrinol Metab. 2007;92(5):1857-62.

26. Yang WS, Lee WJ, Funahashi T, Tanaka S, Matsuzawa Y, Chao CL, et al. Weight reduction increases plasma levels of an adipose-derived anti-inflammatory protein, adiponectin. J Clin Endocrinol Metab. 2001;86(8):3815-9.
27. Flechtner-Mors M, George SN, Oeztuerk S, Haenle MM, Koenig W, Imhof A, et al. Association of adiponectin with hepatic steatosis: a study of 1,349 subjects in a random population sample. BMC Res Notes. 2014;7:207.

28. Akcam M, Boyaci A, Pirgon O, Koroglu M, Dundar BN. Importance of the liver ultrasound scores in pubertal obese children with nonalcoholic fatty liver disease. Clin Imaging. 2013;37(3):504-8.

29. Bydder GM, Chapman RW, Harry D, Bassan L, Sherlock S, Kreel L. Computed tomography attenuation values in fatty liver. J Comput Tomogr. 1981;5(1):33-5.

30. Di MM, Pacifico L, Bezzi M, Di MR, Sacconi B, Chiesa C, et al. Comparison of magnetic resonance spectroscopy, proton density fat fraction and histological analysis in the quantification of liver steatosis in children and adolescents. World J Gastroenterol. 2016;22(39):8812-9.

31. Fishbein MH, Gardner KG, Potter CJ, Schmalbrock P, Smith MA. Introduction of fast MR imaging in the assessment of hepatic steatosis. Magn Reson Imaging. 1997:15(3):287-93.

32. Idilman IS, Keskin O, Celik A, Savas B, Halil EA, Idilman R, et al. A comparison of liver fat content as determined by magnetic resonance imaging-proton density fat fraction and MRS versus liver histology in non-alcoholic fatty liver disease. Acta Radiol. 2016;57(3):271-8.

33. Fitzpatrick E, Quaglia A, Vimalesvaran S, Basso MS, Dhawan A. Transient elastography is a useful noninvasive tool for the evaluation of fibrosis in paediatric chronic liver disease. J Pediatr Gastroenterol Nutr. 2013:56(1):72-6.

34. Kumar R, Rastogi A, Sharma MK, Bhatia V, Tyagi P, Sharma P, et al. Liver stiffness measurements in patients with different stages of nonalcoholic fatty liver disease: diagnostic performance and clinicopathological correlation. Dig Dis Sci. 2013;58(1):265-74.

\section{Submit your next manuscript to BioMed Central and we will help you at every step:}

- We accept pre-submission inquiries

- Our selector tool helps you to find the most relevant journal

- We provide round the clock customer support

- Convenient online submission

- Thorough peer review

- Inclusion in PubMed and all major indexing services

- Maximum visibility for your research

Submit your manuscript at www.biomedcentral.com/submit
) Biomed Central 